\title{
ON LOCAL UNIFORM EQUICONVERGENCE RATE FOR THE DIRAC OPERATOR
}

\author{
VALI M. KURBANOV AND AFSANA M. ABDULLAYEVA
}

\begin{abstract}
We consider one-dimensional Dirac operator with a summable potential on the interval $G=(0,2 \pi)$. Componentwise uniform equiconvergence rate of spectral expansion for absolutely continuous function and trigonometric Fourier series is estimated on a compact.
\end{abstract}

\section{Introduction and statement of results}

In [2-3], Il'in developed a method for studying uniform equiconvergence of spectral expansions for differential operators.

Il'in modified his method in [4] to establish a componentwise uniform equiconvergence in case of Schrodinger operator with a matrix potential. Further, componentwise equiconvergence for an arbitrary order differential operator was established in [5], while componentwise equiconvergence rate was studied in [6].

Componentwise uniform equiconvergence of spectral expansions for Dirac operator was considered in [7], [8]. In [1], componentwise equiconvergence of spectral expansion in the metrics $L_{s}, s \geq 1$, was studied in the compact and a sufficient condition providing equiconvergence in this metrics was found.

In this work, we study componentwise uniform equiconvergence rate of spectral expansion for absolutely continuous vector function with respect to eigenvector functions of Dirac operator and trigonometric Fourier series expansion of this function. We establish estimates for uniform equiconvergence rate on any compact $K \subset G=(0,2 \pi)$.

Consider one-dimensional Dirac operator

$$
D y=B y^{\prime}+P(x) y, \quad y(x)=\left(y_{1}(x), y_{2}(x)\right)^{T},
$$

on the interval $G=(0,2 \pi)$, where $B=\left(\begin{array}{cc}0 & 1 \\ -1 & 0\end{array}\right), P(x)=\left(\begin{array}{cc}p(x) & 0 \\ 0 & q(x)\end{array}\right), p(x)$ and $q(x)$ are real-valued functions from the class $L_{\alpha}(G), \alpha \geq 1$.

Following Il'in [2-4], by the eigenvector function of the operator $D$, corresponding to the real eigenvalue $\lambda$, we mean any not identically zero vector function $y(x)$ which is absolutely continuous on $\bar{G}=[0,2 \pi]$ and satisfies the equation $D y=\lambda y$ almost everywhere in $G$.

2010 Mathematics Subject Classification. 34L10, 42A20.

Key words and phrases. Dirac operator, eigenfunction, equiconvergence, spectral expansion. 
Let $L_{p}^{2}(G), p \geq 1$, be a space of two-component vector functions $f(x)=$ $\left(f_{1}(x), f_{2}(x)\right)^{T}$ with the norm

$$
\|f\|_{p, 2, G}=\|f\|_{p, 2}=\left(\int_{G}|f(x)|^{p} d x\right)^{1 / p}, \quad\left(\|f\|_{\infty, 2}=\sup _{x \in \bar{G}} \operatorname{vrai}|f(x)|\right) .
$$

Obviously, for $f(x) \in L_{p}^{2}(G), g(x) \in L_{q}^{2}(G), p^{-1}+q^{-1}=1, p \geq 1$, there exists a "scalar product"

$$
(f, g)=\int_{G}\langle f(x), g(x)\rangle d x=\int_{G} \sum_{j=1}^{2} f_{j}(x) \overline{g_{j}(x)} d x .
$$

Let $\left\{u_{n}(x)\right\}_{n=1}^{\infty}$ be a complete orthonormal system of eigenvector functions of the operator $D$ in $L_{2}^{2}(G)$ and $\left\{\lambda_{n}\right\}_{n=1}^{\infty}, \lambda_{n} \in R$, be a corresponding system of eigenvalues.

By $W_{p, 2}^{1}(G), p \geq 1$ we denote a space of absolutely continuous two-component vector functions $f(x)$ on $\bar{G}$ with $f^{\prime}(x) \in L_{p}^{2}(G)$.

Introduce partial sum of spectral expansion of the vector function $f(x) \in$ $W_{1,2}^{1}(G)$ with respect to the system $\left\{u_{n}(x)\right\}_{n=1}^{\infty}$ :

$$
\begin{gathered}
\sigma_{\nu}(x, f)=\left(\sigma_{\nu}^{1}(x, f), \sigma_{\nu}^{2}(x, f)\right)^{T}, \sigma_{\nu}^{j}(x, f)=\sum_{\left|\lambda_{n}\right| \leq \nu}\left(f, u_{n}\right) u_{n}^{j}(x), j=1,2 ; \\
\left(u_{n}^{1}(x), u_{n}^{2}(x)\right)^{T}=u_{n}(x), \quad f(x)=\left(f_{1}(x), f_{2}(x)\right)^{T} .
\end{gathered}
$$

In addition to the partial sum $\sigma_{\nu}^{j}(x, f)$ we also introduce a modified partial sum of trigonometric Fourier series of the function $f_{j}(x)$, i.e.

$$
\begin{gathered}
S_{\nu}\left(x, f_{j}\right)=\frac{1}{\pi} \int_{G} \frac{\sin \nu(x-y)}{x-y} f_{j}(y) d y, \quad j=1,2 ; \\
S_{\nu}(x, f)=\left(S_{\nu}\left(x, f_{1}\right), S_{\nu}\left(x, f_{2}\right)\right)^{T} .
\end{gathered}
$$

Definition 1.1. If the difference $\left\|\sigma_{\nu}^{j}(\cdot, f)-S_{\nu}\left(\cdot, f_{j}\right)\right\|_{C(K)}$ tends to zero as $\nu \rightarrow+\infty$ on the compact $K \subset G$, then we say that the $j-$ th component of spectral expansion of the vector function $f(x)$ with respect to the system $\left\{u_{n}(x)\right\}_{n=1}^{\infty}$ uniformly equiconverges on the compact $K \subset G$ with expansion into trigonometric Fourier series corresponding to the $j$-th component $f_{j}(x)$ of the vector function $f(x)$.

The following theorems are the main results of this work.

Theorem 1.1. Let $f(x) \in W_{1,2}^{1}(G)$, the coefficients $p(x)$ and $q(x)$ belong to $L_{\alpha}(G), \alpha>1$. Then the $j-$ th component of spectral expansion of the vector function $f(x)$ with respect to the system $\left\{u_{n}(x)\right\}_{n=1}^{\infty}$ uniformly equiconverges on any compact $K \subset G$ with expansion into trigonometric Fourier series corresponding to the $j$-th component $f_{j}(x)$ of the vector function $f(x)$, and the estimate

$$
\left\|\sigma_{\nu}^{j}(\cdot, f)-S_{\nu}\left(\cdot, f_{j}\right)\right\|_{C(K)}=\left\{\begin{array}{l}
O\left(\nu^{1 / \alpha-1} \ln \nu\right) \text { for } \alpha \in(1, \infty), \\
O\left(\nu^{-1} \ln ^{2} \nu\right) \text { for } \alpha=\infty,
\end{array}\right.
$$

is true as $\nu \rightarrow+\infty$. 
Theorem 1.2. Let the condition

$$
\left.\left\langle B u_{n}(x), f(x)\right\rangle\right|_{0} ^{2 \pi}=0, \quad n=1,2, \ldots,
$$

be fulfilled for the vector function $f(x) \in W_{p, 2}^{1}(G), p>1$, and for the system $\left\{u_{n}(x)\right\}_{n=1}^{\infty}$ and the coefficients $p(x)$ and $q(x)$ belong to $L_{\alpha}(G), \alpha>1$. Then the estimation

$$
\left\|\sigma_{\nu}^{j}(\cdot, f)-S_{\nu}\left(\cdot, f_{j}\right)\right\|_{C(K)}= \begin{cases}O\left(\nu^{1 / \alpha-1}\right) & \text { for } \alpha \in(1, \infty) \\ O\left(\nu^{-1} \ln \nu\right) & \text { for } \alpha=\infty\end{cases}
$$

is true as $\nu \rightarrow+\infty$.

\section{Some auxiliary facts}

Lemma 2.1. ([9]). If the functions $p(x)$ and $q(x)$ belong to the class $L_{1}(G)$ and the points $x-t, x, x+t$ lie on the domain $\bar{G}$, then the following formulas are valid:

$$
\begin{aligned}
& u_{n}(x \pm t)=\left(\cos \lambda_{n} t \cdot I \mp \sin \lambda_{n} t \cdot B\right) u_{n}(x) \pm \int_{x}^{x \pm t}\left\{\sin \lambda_{n}(t-|x-\xi|) I+\right. \\
&\left.+\operatorname{sign}(\xi-x) \cos \lambda_{n}(t-|x-\xi|) B\right\} P(\xi) u_{n}(\xi) d \xi \\
& \frac{u_{n}(x-t)+u_{n}(x-t)}{2}=u_{n}(x) \cos \lambda_{n} t+\frac{1}{2} \int_{x-t}^{x+t}\left\{\sin \lambda_{n}(t-|x-\xi|) I+\right. \\
&\left.+\operatorname{sign}(\xi-x) \cos \lambda_{n}(t-|x-\xi|) B\right\} P(\xi) u_{n}(\xi) d \xi
\end{aligned}
$$

where $I$ is a unit operator in $E^{2}$.

Lemma 2.2. Let the functions $p(x)$ and $q(x)$ belong to the class $L_{1}(G)$. Then there exist the constants $C_{1}$ and $C_{2}$ such that the inequalities

$$
\begin{gathered}
\left\|u_{n}\right\|_{\infty, 2} \leq C_{1}, \quad n=1,2, \ldots, \\
\sum_{\left|t-\lambda_{n}\right| \leq 1} 1 \leq C_{2}, \quad \forall t \in R .
\end{gathered}
$$

are valid.

The estimate (2.3) follows from Theorem 2 of [10], and the estimate (2.4) was proved in [11] (see Theorem $1.4[11]$ ).

Denote

$$
\begin{gathered}
T_{n}^{1}(r, R, \nu)=\int_{r}^{R} \frac{\sin \nu t}{t} \sin \lambda_{n}(t-r) d r \\
T_{n}^{2}(r, R, \nu)=\int_{r}^{R} \frac{\sin \nu t}{t} \cos \lambda_{n}(t-r) d r \\
\left\|T_{n}^{j}(\cdot, R, \nu)\right\|_{p,[0, R]}=\left\{\int_{0}^{R}\left|T_{n}^{j}(r, R, \nu)\right|^{p} d r\right\}^{1 / p},
\end{gathered}
$$


where $R_{0} / 2 \leq R \leq R_{0}, 0<r<R, \nu>0, n \in N, j=1,2 ; p \in[1, \infty], R_{0}$ is a sufficiently small positive number.

The following estimates are valid for the integrals $T_{n}^{j}(r, R, \nu), j=1,2$.

Lemma 2.3. The estimates

$$
\begin{gathered}
\left|T_{n}^{j}(r, R, \nu)\right| \leq C(\beta)\left\{\begin{array}{l}
|\nu-| \lambda_{n}||^{-\beta} r^{-\beta} \text { for }|\nu-| \lambda_{n}|| \geq 1, \\
\max \{|\ln r|,|\ln R|\}, \text { for }|\nu-| \lambda_{n}||<1,
\end{array}\right. \\
\left\|T_{n}^{j}(\cdot, R, \nu)\right\|_{\gamma,[0, R]} \leq C\left(R_{0}\right)\left\{\begin{array}{l}
|\nu-| \lambda_{n}||^{-\frac{1}{\gamma}} \text { for }|\nu-| \lambda_{n}|| \geq 1, \\
1, \quad \text { for }|\nu-| \lambda_{n}||<1,
\end{array}\right. \\
\qquad C T_{n}^{j}(\cdot, R, \nu) \|_{1,[0, R]} \leq \\
\leq C\left(R_{0}\right)\left\{\begin{array}{l}
\left.|\nu-| \lambda_{n}\right|^{-1}\left(1+\ln |\nu-| \lambda_{n}||\right) \text { for }\left|\nu-\lambda_{n}\right| \geq 1, \\
1 \quad \text { for }|\nu-| \lambda_{n}||<1,
\end{array}\right.
\end{gathered}
$$

are valid for the integrals $T_{n}^{j}(r, R, \nu), j=1,2 ; n \in N$, with any $\beta \in(0,1]$ where $\gamma \in(1, \infty)$.

Proof. The estimates (2.5) and (2.6) were proved in [1]. Let us prove (2.7). Fix the number $R_{0}, 0<R_{0} \leq \frac{1}{2}$ and consider the case $R_{0}|\nu-| \lambda_{n}|| \geq 2$. Then $|\nu-| \lambda_{n}||^{-1} \leq R_{0} / 2 \leq R$. By the triangle inequality, we have

$$
\left\|T_{n}^{j}(\cdot, R, \nu)\right\|_{1,[0, R]} \leq\left\|T_{n}^{j}(\cdot, R, \nu)\right\|_{1,\left[0,|\nu-| \lambda_{n} \|^{-1}\right]}+\left\|T_{n}^{j}(\cdot, R, \nu)\right\|_{1,\left[|\nu-| \lambda_{n} \|^{-1}, R\right]} .
$$

Apply the estimate (2.5) to the first term on the right-hand side of this inequality with $|\nu-| \lambda_{n}|| \geq \frac{2}{R_{0}} \geq 1, \beta=\frac{1}{2}$, and to the second term with $\beta=1$. Then we obtain

$$
\begin{gathered}
\left\|T_{n}^{j}(\cdot, R, \nu)\right\|_{1,\left[0,\left.|\nu-| \lambda_{n}\right|^{-1}\right]}=O\left(\int_{0}^{\left.|\nu-| \lambda_{n}\right|^{-1}}|\nu-| \lambda_{n}||^{-\frac{1}{2}} r^{-\frac{1}{2}} d r\right)= \\
=O\left(|\nu-| \lambda_{n}||^{-\frac{1}{2}}\right)\left(|\nu-| \lambda_{n}||^{-\frac{1}{2}}\right)=O\left(|\nu-| \lambda_{n}||^{-1}\right) ; \\
\left\|T_{n}^{j}(\cdot, R, \nu)\right\|_{1,\left[\left.|\nu-| \lambda_{n}\right|^{-1}, R\right]}=O\left(\int_{\nu-\left|\lambda_{n}\right|^{-1}}^{R}|\nu-| \lambda_{n}||^{-1} r^{-1} d r\right)= \\
=O\left(\left.|\nu-| \lambda_{n}\right|^{-1}\right)\left(\int_{\nu-\left|\lambda_{n}\right|^{-1}}^{R} r^{-1} d r\right)= \\
=O\left(\left.|\nu-| \lambda_{n}\right|^{-1}\right)\left(\ln R_{0}+\ln |\nu-| \lambda_{n}||\right)= \\
=O\left(|\nu-| \lambda_{n}||^{-1}\right)\left(1+\ln |\nu-| \lambda_{n}||\right) .
\end{gathered}
$$

If $1 \leq|\nu-| \lambda_{n}||<\frac{2}{R_{0}}$, then, applying inequality (2.5) with $\beta=\frac{1}{2}$, we get

$$
\begin{gathered}
\left\|T_{n}^{j}(\cdot, R, \nu)\right\|_{1,[0, R]}=O\left(|\nu-| \lambda_{n}||^{-\frac{1}{2}}\right) R_{0}^{1 / 2}= \\
=O\left(|\nu-| \lambda_{n}||^{-\frac{1}{2}}\right)\left(\frac{2}{|\nu-| \lambda_{n}||}\right)^{1 / 2}=O\left(|\nu-| \lambda_{n}||^{-1}\right) .
\end{gathered}
$$


For $|\nu-| \lambda_{n}||<1$, the estimate (2.7) follows from (2.5) taking into account the integrability of the function $|\ln r|$ on $[0, R]$. So we get the validity of $(2.7)$.

Lemma 2.4. If $\left|\lambda_{n}\right| \geq 1$ then the estimate

$$
\left|f_{n}\right| \leq \frac{C(f)}{\left|\lambda_{n}\right|}+\frac{1}{\left|\lambda_{n}\right|}\left|\left(B^{*} f^{\prime}, u_{n}\right)\right|+\frac{1}{\left|\lambda_{n}\right|}\left|\left(P f, u_{n}\right)\right|,
$$

is valid for the Fourier coefficients of the arbitrary vector function $f(x) \in W_{1,2}^{1}(G)$ with respect to the system $\left\{u_{n}(x)\right\}_{n=1}^{\infty}$, where $C(f)$ is a positive constant.

Proof. From the equation $D u_{n}=\lambda_{n} u_{n}$ for $\left(u_{n}, f\right)$ we get

$$
\begin{aligned}
& \left(u_{n}, f\right)=\int_{0}^{2 \pi}\left\langle u_{n}(x), f(x)\right\rangle d x=\frac{1}{\lambda_{n}} \int_{0}^{2 \pi}\left\langle D u_{n}(x), f(x)\right\rangle d x= \\
& =\frac{1}{\lambda_{n}} \int_{0}^{2 \pi}\left\langle B u_{n}^{\prime}(x), f(x)\right\rangle d x+\frac{1}{\lambda_{n}} \int_{0}^{2 \pi}\left\langle P(x) u_{n}(x), f(x)\right\rangle d x .
\end{aligned}
$$

Integrating by parts, from the last relation we obtain

$$
\begin{gathered}
\left(u_{n}, f\right)=\left.\frac{1}{\lambda_{n}}\left\langle B u_{n}(x), f(x)\right\rangle\right|_{0} ^{2 \pi}-\frac{1}{\lambda_{n}} \int_{0}^{2 \pi}\left\langle B u_{n}(x), f^{\prime}(x)\right\rangle d x+ \\
+\frac{1}{\lambda_{n}} \int_{0}^{2 \pi}\left\langle P(x) u_{n}(x), f(x)\right\rangle d x=\left.\frac{1}{\lambda_{n}}\left(\overline{f_{1}(x)} u_{n 2}(x)-\overline{f_{2}(x)} u_{n 1}(x)\right)\right|_{0} ^{2 \pi}- \\
-\frac{1}{\lambda_{n}} \int_{0}^{2 \pi}\left\langle u_{n}(x), B^{*} f^{\prime}(x)\right\rangle d x+\frac{1}{\lambda_{n}} \int_{0}^{2 \pi}\left\langle u_{n}(x), P^{*}(x) f(x)\right\rangle d x,
\end{gathered}
$$

where $B^{*}=\left(\begin{array}{cc}0 & -1 \\ 1 & 0\end{array}\right), P^{*}(x)=P(x), u_{n}(x)=\left(u_{n 1}(x), u_{n 2}(x)\right)^{T}$.

By (2.3), we have

$$
\begin{gathered}
\left|\left(\overline{f_{1}(x)} u_{n 2}(x)-\overline{f_{2}(x)} u_{n 1}(x)\right)\right| \begin{array}{l}
2 \pi \\
0
\end{array} \mid \leq \\
\leq C_{1}\left(\left|f_{1}(2 \pi)\right|+\left|f_{1}(0)\right|+\left|f_{2}(2 \pi)\right|+\left|f_{2}(0)\right|\right) \equiv C(f) .
\end{gathered}
$$

Using this inequality, from (2.9) we get the estimate (2.8). Lemma 2.4 is proved.

Corollary 2.1. If the vector function $f(x) \in W_{1,2}^{1}(G)$ and the system $\left\{u_{n}(x)\right\}_{n=1}^{\infty}$ satisfy the condition (1.2), then the following estimate is valid for the coefficients $f_{n}=\left(f, u_{n}\right)$ with $\left|\lambda_{n}\right| \geq 1$ :

$$
\left|f_{n}\right| \leq\left|\lambda_{n}\right|^{-1}\left\{\left|\left(B^{*} f^{\prime}, u_{n}\right)\right|+\left|\left(P f, u_{n}\right)\right|\right\} .
$$

In particular, (2.10) is valid for the Fourier coefficients $f_{n}$ of the vector function $f(x) \in W_{1,2}^{1}(G)$ satisfying the condition $f(0)=f(2 \pi)=0$. 
Lemma 2.5. $\left\{u_{n}(x)\right\}_{n=1}^{\infty}$ is a Riesz system, i.e. the Riesz inequality

$$
\left(\sum_{n=1}^{\infty}\left|\left(f, u_{n}\right)\right|^{q}\right)^{1 / q} \leq M_{p}\|f\|_{p, 2}
$$

holds for every vector function $f(x) \in L_{p}^{2}(G), 1<p \leq 2$, where $M_{p}>0$ is a constant independent of $f(x), p^{-1}+q^{-1}=1$.

The proof of Lemma 2.5 follows from the orthonormality and uniform boundedness of the system $\left\{u_{n}(x)\right\}_{n=1}^{\infty}$ (see (2.3)) and from the Riesz theorem [13. p. $154]$.

\section{Proof of results}

Proof of theorem 1.1. Let $f(x)$ be an arbitrary function from $W_{1,2}^{1}(G)$ and $p(x), q(x) \in L_{\alpha}(G)$. Fixing an arbitrary connected compact $K \subset G$, we choose the number $R_{0}$ satisfying the condition $0 \leq 4 R_{0}<\min \{1, \operatorname{dist}(K, \partial G)\}$. Let us introduce the following vector function:

$$
\tilde{S}_{\nu}(x, f)=\left(\tilde{S}_{\nu}\left(x, f_{1}\right), \tilde{S}_{\nu}\left(x, f_{2}\right)\right)^{T},
$$

where $\tilde{S}_{\nu}\left(x, f_{j}\right), j=1,2$, is is defined by the formula

$$
\begin{gathered}
\tilde{S}_{\nu}\left(x, f_{j}\right)=\frac{1}{\pi} \int_{|x-y| \leq R} \frac{\sin \nu(x-y)}{x-y} f_{j}(y) d y, \quad j=1,2, \\
x \in K, \quad R \in\left[R_{0} / 2, R_{0}\right], \quad f(x)=\left(f_{1}(x), f_{2}(x)\right)^{T} .
\end{gathered}
$$

As the difference $S_{\nu}\left(x, f_{j}\right)-\tilde{S}_{\nu}\left(x, f_{j}\right)$ tends to zero uniformly with respect to $x \in K$ with $\nu \rightarrow+\infty$ and is of order $O\left(\nu^{-1}\right)$ (see [12]), it suffices to prove Theorem 1.1 for the partial sum $\tilde{S}_{\nu}(x, f)$.

As $W_{1,2}^{1}(G) \subset L_{2}^{2}(G)$ and the system $\left\{u_{n}(x)\right\}_{n=1}^{\infty}$ forms a basis for $L_{2}^{2}(G)$, every vector function $f(x) \in W_{1,2}^{1}(G)$ can be represented in the form

$$
f(x)=\sum_{n=1}^{\infty} f_{n} u_{n}(x), \quad f_{n}=\left(f, u_{n}\right)
$$

where the convergence of the series is to be understood in the norm of the space $L_{2}^{2}(G)$. Therefore, the partial sum $\tilde{S}_{\nu}(x, f)$ can be represented as

$$
\tilde{S}_{\nu}(x, f)=\frac{2}{\pi} \sum_{n=1}^{\infty}\left(f, u_{n}\right) \int_{0}^{R} \frac{\sin \nu t}{t} \frac{u_{n}(x-t)+u_{n}(x+t)}{2} d t .
$$

Applying the mean value formula (2.2) and carrying out some calculations (see [1] for details), we get

$$
\tilde{S}_{\nu}(x, f)-\sigma_{\nu}(x, f)=-\frac{1}{2} \sum_{\left|\lambda_{n}\right|=\nu}\left(f, u_{n}\right) u_{n}(x)+\sum_{n=1}^{\infty}\left(f, u_{n}\right) u_{n}(x) I\left(\nu, \lambda_{n}\right)+
$$




$$
\begin{gathered}
+\frac{1}{\pi} \sum_{n=1}^{\infty}\left(f, u_{n}\right) \int_{0}^{R}\left\{P(x+r) u_{n}(x+r)+P(x-r) u_{n}(x-r)\right\} \times \\
\times T_{n}^{1}(r, R, \nu) d r+\frac{1}{\pi} \sum_{n=1}^{\infty}\left(f, u_{n}\right) \times \\
\times \int_{0}^{R}\left\{P(x+r) u_{n}(x+r)-P(x-r) u_{n}(x-r)\right\} T_{n}^{2}(r, R, \nu) d r= \\
=\Phi_{1}(x, f)+\Phi_{2}(x, f)+\Phi_{3}(x, f)+\Phi_{4}(x, f),
\end{gathered}
$$

where $x \in K$, and the estimate

$$
\left|I\left(\nu, \lambda_{n}\right)\right| \leq C(R)\left(1+|\nu-| \lambda_{n}||\right)^{-1} .
$$

is valid for the factors $I\left(\nu, \lambda_{n}\right)$ (see [2-3], [1])

Let us estimate the series $\Phi_{j}(x, f), j=1,4$ in the metrics $C(K)$. To estimate the series $\Phi_{1}(x, f)$, we use Lemma 2.2 and the estimate (2.8):

$$
\begin{gathered}
\left\|\Phi_{1}(\cdot, f)\right\|_{C(K)}=\frac{1}{2}\left\|\sum_{\left|\lambda_{n}\right|=\nu}\left(f, u_{n}\right) u_{n}\right\|_{C(K)} \leq \\
\leq C_{1} \sum_{\left|\lambda_{n}\right|=\nu}\left|\left(f, u_{n}\right)\right| \leq C_{1} C(f) \sum_{\left|\lambda_{n}\right|=\nu}\left|\lambda_{n}\right|^{-1}+ \\
+C_{1}\left\|B^{*} f^{\prime}\right\|_{1,2} \sum_{\left|\lambda_{n}\right|=\nu}\left|\lambda_{n}\right|^{-1}\left\|u_{n}\right\|_{\infty, 2}+C_{1}\|P f\|_{1,2} \sum_{\left|\lambda_{n}\right|=\nu}\left|\lambda_{n}\right|^{-1}\left\|u_{n}\right\|_{\infty, 2} \leq \\
\leq C_{1} C(f) \nu^{-1}\left(\sum_{\left|\lambda_{n}\right|=\nu} 1\right)+C_{1}^{2}\left\|B^{*} f^{\prime}\right\|_{1,2} \nu^{-1}\left(\sum_{\left|\lambda_{n}\right|=\nu} 1\right)+ \\
+C_{1}^{2}\|P f\|_{1,2} \nu^{-1}\left(\sum_{\left|\lambda_{n}\right|=\nu} 1\right) \leq \\
\leq\left(C_{1} C_{2} C(f)+C_{2} C_{1}^{2}\left\|B^{*} f^{\prime}\right\|_{1,2}+C_{2} C_{1}^{2}\|P f\|_{1,2}\right) \nu^{-1} \leq C_{1}(f) \nu^{-1} .
\end{gathered}
$$

Consequently, the following estimate is true for the sum $\Phi_{1}(x, f)$

$$
\left\|\Phi_{1}(\cdot, f)\right\|_{C(K)}=O\left(\nu^{-1}\right) .
$$

Let us estimate the series $\Phi_{2}(x, f)$. To do so, we apply the inequalities (2.3), (3.2) and make use of the estimate (2.8) of Lemma 2.4. Then we have

$$
\begin{gathered}
\left\|\Phi_{2}(\cdot, f)\right\|_{C(K)}=\left\|\sum_{n=1}^{\infty}\left(f, u_{n}\right) u_{n}(x) I\left(\nu, \lambda_{n}\right)\right\|_{C(K)} \leq \\
\leq C(R) C_{1}(f) \sum_{\left|\lambda_{n}\right| \geq 1}^{\infty}\left|\lambda_{n}\right|^{-1}\left(1+|\nu-| \lambda_{n}||\right)^{-1}+ \\
+C_{1}^{2} C(R)\|f\|_{1,2} \sum_{\left|\lambda_{n}\right| \leq 1}\left(1+|\nu-| \lambda_{n}||\right)^{-1} \leq
\end{gathered}
$$




$$
\begin{aligned}
& \leq C(R) C_{1}(f)\left\{\sum_{\substack{|| \lambda_{n}|-\nu| \leq 1,\left|\lambda_{n}\right| \geq 1\\
}}\left|\lambda_{n}\right|^{-1}\left(1+|\nu-| \lambda_{n}||\right)^{-1}+\right. \\
& +\sum_{\substack{1 \leq|| \lambda_{n}|-\nu| \leq \nu / 2 \\
\left|\lambda_{n}\right| \geq 1}}\left|\lambda_{n}\right|^{-1}\left(1+|\nu-| \lambda_{n}||\right)^{-1}+ \\
& \left.+\sum_{\substack{|| \lambda_{n}|-\nu| \geq \nu / 2 \\
\left|\lambda_{n}\right| \geq 1}}\left|\lambda_{n}\right|^{-1}\left(1+|| \lambda_{n}|-\nu|\right)^{-1}\right\}+C_{1}^{2} C(R) \nu^{-1}\|f\|_{1,2} \leq \\
& \leq C(R) C_{1}(f)\left\{\sum_{|| \lambda_{n}|-\nu| \leq 1,\left|\lambda_{n}\right| \geq 1}\left|\lambda_{n}\right|^{-1}+\left.\sum_{1 \leq|| \lambda_{n}|-\nu| \leq \nu / 2,\left|\lambda_{n}\right| \geq 1}\left|\lambda_{n}\right|^{-1}|\nu-| \lambda_{n}\right|^{-1}+\right. \\
& \left.+\sum_{|| \lambda_{n}|-\nu| \geq \nu / 2,\left|\lambda_{n}\right| \geq 1}\left|\lambda_{n}\right|^{-1}\left(1+|\nu-| \lambda_{n}||\right)^{-1}\right\}+O\left(\nu^{-1}\right) .
\end{aligned}
$$

The first term inside curly braces has been already estimated above and is of order $O\left(\nu^{-1}\right)$. The two remaining series, by virtue of inequality (2.4), can be estimated as follows:

$$
\begin{aligned}
& \left.\sum_{1 \leq|| \lambda_{n}|-\nu| \leq \nu / 2,\left|\lambda_{n}\right| \geq 1}\left|\lambda_{n}\right|^{-1}|\nu-| \lambda_{n}\right|^{-1} \leq \frac{2}{\nu} \sum_{1 \leq|| \lambda_{n}|-\nu| \leq \nu / 2}|| \lambda_{n}|-\nu|^{-1} \leq \\
& \leq \frac{2}{\nu} \sum_{k=1}^{[\nu / 2]} k^{-1}\left(\sum_{k \leq|| \lambda_{n}|-\nu| \leq k+1} 1\right) \leq \frac{2 C_{1}}{\nu} \sum_{k=1}^{[\nu / 2]} k^{-1}=O\left(\frac{\ln \nu}{\nu}\right) ; \\
& \sum_{|| \lambda_{n}|-\nu| \geq \nu / 2,\left|\lambda_{n}\right| \geq 1}\left|\lambda_{n}\right|^{-1}\left(1+|| \lambda_{n}|-\nu|\right)^{-1}=\sum_{1 \leq\left|\lambda_{n}\right| \leq \nu / 2}\left|\lambda_{n}\right|^{-1}\left(|| \lambda_{n}|-\nu|+1\right)^{-1}+ \\
& +\sum_{\left|\lambda_{n}\right| \geq 3 \nu / 2}\left|\lambda_{n}\right|^{-1}\left(1+|| \lambda_{n}|-\nu|\right)^{-1} \leq(1+\nu / 2)^{-1} \sum_{1 \leq\left|\lambda_{n}\right| \leq \nu / 2}\left|\lambda_{n}\right|^{-1}+ \\
& +\sum_{\left|\lambda_{n}\right| \geq 3 \nu / 2}|| \lambda_{n}|-\nu|^{-2}=O\left(\frac{\ln \nu}{\nu}\right)+O\left(\nu^{-1}\right)=O\left(\frac{\ln \nu}{\nu}\right) .
\end{aligned}
$$

Thus, the following estimate is true for the sum $\Phi_{2}(x, f)$

$$
\left\|\Phi_{2}(\cdot, f)\right\|_{C(K)}=O\left(\frac{\ln \nu}{\nu}\right) .
$$

Now let us estimate the series $\Phi_{3}(x, f)$. By virtue of (2.3)

$$
\begin{gathered}
\left|\Phi_{3}(x, f)\right|=\frac{1}{\pi} \mid \sum_{n=1}^{\infty}\left(f, u_{n}\right) \int_{0}^{R}\left\{P(x+r) u_{n}(x+r)+\right. \\
\quad+P(x-r) u\left({ }_{n}(x-r)\right\} T_{n}^{1}(r, R, \nu) d r \mid \leq \\
\leq C \sum_{n=1}^{\infty}\left|\left(f, u_{n}\right)\right| \int_{0}^{R} Q(x, r)\left|T_{n}^{1}(r, R, \nu)\right| d r,
\end{gathered}
$$


where $Q(x, r)=|p(x+r)|+|p(x-r)|+|q(x-r)|+|q(x+r)|$.

Introduce the following integrals

$$
L^{ \pm}(x)=\int_{0}^{R}|p(x \pm r)|\left|T_{n}^{1}(r, R, \nu)\right| d r ; M^{ \pm}(x)=\int_{0}^{R}|q(x \pm r)|\left|T_{n}^{1}(r, R, \nu)\right| d r .
$$

By Hölder's inequality, we have

$$
\left\|L^{ \pm}\right\|_{C(K)} \leq\|p\|_{\alpha}\left\|T_{n}^{1}(\cdot, R, \nu)\right\|_{\alpha^{\prime},[0, R]},
$$

where $1 / \alpha+1 / \alpha^{\prime}=1$.

Using here lemma 2.3 (see estimations $(2.6),(2.7)$ ), we get

$$
\begin{gathered}
\left\|L^{ \pm}\right\|_{C(K)} \leq C\left(R_{0}\right)\|p\|_{\alpha} \times \\
\times\left\{\begin{array}{l}
|\nu-| \lambda_{n}||^{-1 / \alpha^{\prime}} \text { for }|\nu-| \lambda_{n}|| \geq 1, \alpha \in(1, \infty), \\
1 \quad \text { for }|\nu-| \lambda_{n}||<1, \\
|\nu-| \lambda_{n}||^{-1}\left(\ln |\nu-| \lambda_{n}||+1\right), \text { for }|\nu-| \lambda_{n}|| \geq 1, \alpha=\infty .
\end{array}\right.
\end{gathered}
$$

Obviously, the same estimate holds for the integrals $M^{ \pm}(x)$, i.e.

$$
\begin{gathered}
\left\|M^{ \pm}\right\|_{C(K)} \leq C\left(R_{0}\right)\|q\|_{\alpha} \times \\
\times\left\{\begin{array}{l}
1 \text { for }|\nu-| \lambda_{n}||<1, \\
|\nu-| \lambda_{n}||^{-1 / \alpha^{\prime}} \text { for }|\nu-| \lambda_{n}|| \geq 1, \alpha \in(1, \infty), \\
|\nu-| \lambda_{n}||^{-1}\left(\ln |\nu-| \lambda_{n}||+1\right) \text { for }|\nu-| \lambda_{n}|| \geq 1, \alpha=\infty .
\end{array} .\right.
\end{gathered}
$$

Let $\alpha \in(1, \infty)$. Then from the inequality (3.5) taking into account the estimates (3.6) and (3.7) we get

$$
\begin{gathered}
\left\|\Phi_{3}(\cdot, f)\right\|_{C(K)} \leq C \sum_{n=1}^{\infty}\left|\left(f, u_{n}\right)\right|\left\|\int_{0}^{R} Q(\cdot, r)\left|T_{n}^{1}(r, R, \nu)\right| d r\right\|_{C(K)} \leq \\
\leq C\left(R_{0}\right)\left(\|p\|_{\alpha}+\|q\|_{\alpha}\right) \times \\
\quad \times\left\{\sum_{|\nu-| \lambda_{n}||<1}\left|\left(f, u_{n}\right)\right|+\sum_{|\nu-| \lambda_{n}|| \geq 1}\left|\left(f, u_{n}\right)\right||\nu-| \lambda_{n}||^{-1 / \alpha^{\prime}}\right\}
\end{gathered}
$$

As $\nu>2$, the inequality $\left|\lambda_{n}\right| \geq 1$ is true for $\lambda_{n}$ satisfying $|\nu-| \lambda_{n}||<1$. Therefore, Lemma 2.4 can be applied to estimate the first term inside curly braces. Then,

$$
\begin{aligned}
\sum_{|\nu-| \lambda_{n}||<1}\left|\left(f, u_{n}\right)\right| \leq & C(f) \sum_{|\nu-| \lambda_{n}||<1}\left|\lambda_{n}\right|^{-1}+C_{1}\left\|B^{*} f^{\prime}\right\|_{1,2} \sum_{|\nu-| \lambda_{n}||<1}\left|\lambda_{n}\right|^{-1}+ \\
& +C_{1}\|P f\|_{1,2} \sum_{|\nu-| \lambda_{n} \| \leq 1}\left|\lambda_{n}\right|^{-1} \leq \\
\leq C_{2} & \left(C(f)+C_{1}\left\|B^{*} f^{\prime}\right\|_{1,2}+C_{1}\|P f\|_{1,2}\right) \times \\
& \times(\nu-1)^{-1}=O\left(\nu^{-1}\right), \nu \rightarrow+\infty .
\end{aligned}
$$

Let us divide the second term in curly braces on the right-hand side of (3.8) ) into two sums, the first of them corresponding to the case $|\nu-| \lambda_{n}|| \geq 1,\left|\lambda_{n}\right|<1$, and the second one corresponding to the case $|\nu-| \lambda_{n}|| \geq 1, \quad\left|\lambda_{n}\right| \geq 1$. 
In the first case, we apply the inequality $\left|\left(f, u_{n}\right)\right| \leq\|f\|_{2,2}$ to the coefficients $\left(f, u_{n}\right)$, and in the second case we apply lemma 2.4 and use the estimate (2.4). Then we obtain the following inequalities:

$$
\begin{aligned}
& \sum_{|\nu-| \lambda_{n}|| \geq 1}\left|\left(f, u_{n}\right)\right||\nu-| \lambda_{n}||^{-1 / \alpha^{\prime}} \leq \sum_{\substack{|\nu-| \lambda_{n}|| \geq 1 \\
\left|\lambda_{n}\right|<1}}|\nu-| \lambda_{n}||^{-1 / \alpha^{\prime}}\|f\|_{2,2}+ \\
& +\sum_{\substack{|\nu-| \lambda_{n} \| \geq 1 \\
\left|\lambda_{n}\right| \geq 1}}\left\{C(f)+C_{1}\left\|B^{*} f^{\prime}\right\|_{1,2}+C_{1}\|P f\|_{1,2}\right\}\left|\lambda_{n}\right|^{-1}|\nu-| \lambda_{n} \|^{-1 / \alpha^{\prime}} \leq \\
& \leq\left.\sum_{\left|\lambda_{n}\right|<1}|\nu-| \lambda_{n}\right|^{-1 / \alpha^{\prime}}\|f\|_{2,2}+C_{2}(f) \sum_{\substack{|\nu-| \lambda_{n} \| \geq 1 \\
\left|\lambda_{n}\right| \geq 1}}\left|\lambda_{n}\right|^{-1}|\nu-| \lambda_{n}||^{-1 / \alpha^{\prime}} \leq \\
& \leq(\nu-1)^{-1 / \alpha^{\prime}}\|f\|_{2,2}\left(\sum_{\left|\lambda_{n}\right|<1} 1\right)+C_{2}(f) \sum_{\substack{1 \leq|\nu-| \lambda_{n}|| \leq \nu / 2 \\
\left|\lambda_{n}\right| \geq 1}}\left|\lambda_{n}\right|^{-1}|\nu-| \lambda_{n} \|^{-1 / \alpha^{\prime}}+ \\
& +C_{2}(f) \sum_{\substack{|\nu-| \lambda_{n} \| \geq \nu / 2 \\
\left|\lambda_{n}\right| \geq 1}}\left|\lambda_{n}\right|^{-1}|\nu-| \lambda_{n}\left\|^{-1 / \alpha^{\prime}} \leq C_{2}\right\| f \|_{2,2}\left(1-\nu^{-1}\right)^{-1 / \alpha^{\prime}} \nu^{-1 / \alpha^{\prime}}+ \\
& +2 C_{2}(f) \nu^{-1} \sum_{\substack{1 \leq|\nu-| \lambda_{n} \| \leq \nu / 2 \\
\left|\lambda_{n}\right| \geq 1}}|\nu-| \lambda_{n}||^{-1 / \alpha^{\prime}}+ \\
& +C_{2}(f)\left\{\left.\sum_{1 \leq\left|\lambda_{n}\right| \leq \nu / 2}\left|\lambda_{n}\right|^{-1}|\nu-| \lambda_{n}\right|^{-1 / \alpha^{\prime}}+\left.\sum_{\left|\lambda_{n}\right| \geq 3 \nu / 2}\left|\lambda_{n}\right|^{-1}|\nu-| \lambda_{n}\right|^{-1 / \alpha^{\prime}}\right\} \leq \\
& \leq O\left(\nu^{-1 / \alpha^{\prime}}\right)+O\left(\nu^{-1 / \alpha^{\prime}}\right)+O\left(\nu^{-1 / \alpha^{\prime}} \ln \nu\right)+O\left(\nu^{-1 / \alpha^{\prime}}\right)=O\left(\nu^{-1 / \alpha^{\prime}} \ln \nu\right) .
\end{aligned}
$$

Taking into account this estimate and the estimate (3.9), from (3.8) we obtain

$$
\left\|\Phi_{3}(\cdot, f)\right\|_{C(K)}=O\left(\nu^{-1 / \alpha^{\prime}} \ln \nu\right), \nu \rightarrow \infty .
$$

The series $\Phi_{4}(x, f)$ is estimated in the same way, and the estimate (3.10) is valid for it. Thus, the estimate (3.10) is valid for the series $\Phi_{3}(x, f)$ and $\Phi_{4}(x, f)$ when $\alpha \in(1, \infty)$.

Consider the case $\alpha=\infty$. Applying the estimates (3.6) and (3.7) for $\alpha=\infty$ we get (see (3.8), (3.9))

$$
\begin{gathered}
\left\|\Phi_{3}(\cdot, f)\right\|_{C(K)} \leq C\left(R_{0}\right)\left(\|p\|_{\infty}+\|q\|_{\infty}\right)\left\{\sum_{|\nu-| \lambda_{n}||<1}\left|\left(f, u_{n}\right)\right|+\right. \\
\left.+\sum_{|\nu-| \lambda_{n}|| \geq 1}\left|\left(f, u_{n}\right)\right||\nu-| \lambda_{n}||^{-1} \ln \left(1+|\nu-| \lambda_{n}||\right)\right\} \leq \\
\leq C\left(R_{0}\right)\left(\|p\|_{\infty}+\|q\|_{\infty}\right) O\left(\nu^{-1}\right)+C\left(R_{0}\right)\left(\|p\|_{\infty}+\|q\|_{\infty}\right) \\
\sum_{|\nu-| \lambda_{n} \| \geq 1}\left|\left(f, u_{n}\right)\right||\nu-| \lambda_{n}||^{-1} \ln \left(1+|\nu-| \lambda_{n}||\right) .
\end{gathered}
$$


Let us prove that the second term on the right-hand side of (3.11) is of order $O\left(\nu^{-1} \ln ^{2} \nu\right), \nu \rightarrow+\infty$. For this aim, we divide this sum into two sums, apply the inequality $\left|\left(f, u_{n}\right)\right| \leq\|f\|_{2,2}$ in the case $\left|\lambda_{n}\right|<1,|\nu-| \lambda_{n}|| \geq 1$ and lemma 2.4 in the case $\left|\lambda_{n}\right| \geq 1,|\nu-| \lambda_{n}|| \geq 1$ :

$$
\begin{aligned}
& \sum_{|\nu-| \lambda_{n}|| \geq 1}\left|\left(f, u_{n}\right)\right||\nu-| \lambda_{n}||^{-1} \ln \left(1+|\nu-| \lambda_{n}||\right) \leq \\
& \leq \sum_{\substack{\left|\lambda_{n}\right|<1 \\
|\nu-| \lambda_{n}|| \geq 1}}|\nu-| \lambda_{n}||^{-1} \ln \left(1+|\nu-| \lambda_{n}||\right)\|f\|_{2,2}+ \\
& +\left.C_{2}(f) \sum_{|\nu-| \lambda_{n}|| \geq 1,\left|\lambda_{n}\right| \geq 1}\left|\lambda_{n}\right|^{-1}|\nu-| \lambda_{n}\right|^{-1} \ln \left(1+|\nu-| \lambda_{n}||\right) \leq \\
& \leq(\nu-1)^{-1} \ln \nu\|f\|_{2,2}\left(\sum_{|\nu-| \lambda_{n}||<1} 1\right)+C_{2}(f) \sum_{\substack{1 \leq|\nu-| \lambda_{n}|| \leq \nu / 2 \\
\left|\lambda_{n}\right| \geq 1}}(\cdot)+ \\
& +C_{2}(f) \sum_{\substack{|\nu-| \lambda_{n}|| \geq \nu / 2 \\
\left|\lambda_{n}\right| \geq 1}}(\cdot) \leq O\left(\frac{\ln \nu}{\nu}\right)+2 C_{2}(f) \nu^{-1} \sum_{1 \leq|\nu-| \lambda_{n}|| \leq \nu / 2}|\nu-| \lambda_{n}||^{-1} \\
& \ln \left(1+|\nu-| \lambda_{n}||\right)+C_{2}(f)\left\{\sum_{1 \leq\left|\lambda_{n}\right| \leq \nu / 2}\left|\lambda_{n}\right|^{-1}|\nu-| \lambda_{n}||^{-1} \ln \left(1+|\nu-| \lambda_{n}||\right)+\right. \\
& \left.+\sum_{\left|\lambda_{n}\right| \geq 3 \nu / 2}\left|\lambda_{n}\right|^{-1}|\nu-| \lambda_{n}||^{-1} \ln \left(1+|\nu-| \lambda_{n}||\right)\right\} \leq \\
& \leq O\left(\frac{\ln \nu}{\nu}\right)+O\left(\frac{\ln ^{2} \nu}{\nu}\right)+O\left(\frac{\ln \nu}{\nu}\right)\left(\sum_{1 \leq\left|\lambda_{n}\right| \leq \nu / 2} 1\right)+ \\
& +C_{2}(f) \sum_{|| \lambda_{n}|-\nu| \geq \nu / 2}|\nu-| \lambda_{n}||^{-2} \ln \left(1+|\nu-| \lambda_{n}||\right)=O\left(\frac{\ln ^{2} \nu}{\nu}\right) .
\end{aligned}
$$

Consequently, the following estimate is true for the series $\Phi_{3}(x, f)$ (as well as for the series $\left.\Phi_{4}(x, f)\right)$ when $\alpha=\infty$ (see $\left.(3.11)\right)$ :

$$
\left\|\Phi_{3}(\cdot, f)\right\|_{C(K)}=O\left(\frac{\ln ^{2} \nu}{\nu}\right), \nu \rightarrow+\infty .
$$

Now, taking into account the estimates (3.3), (3.4), (3.10) and (3.12) in (3.1), we get

$$
\max _{x \in K}\left|\sigma_{\nu}(x, f)-\tilde{S}_{\nu}(x, f)\right|= \begin{cases}O\left(\nu^{\frac{1}{\alpha}-1} \ln \nu\right) & \text { for } \alpha \in(1, \infty), \\ O\left(\nu^{-1} \ln ^{2} \nu\right) & \text { for } \alpha=\infty\end{cases}
$$

as $\nu \rightarrow+\infty$. Theorem 1.1 is completely proved.

Proof of theorem 1.2. Let the vector function $f(x) \in W_{p, 2}^{1}(G), p>1$ and the system $\left\{u_{n}(x)\right\}_{n=1}^{\infty}$ satisfy the condition (1.2), i.e. $\left.\left\langle B u_{n}(x), f(x)\right\rangle\right|_{0} ^{2 \pi}=0$. Let us estimate the difference $\tilde{S}_{\nu}(x, f)-\sigma_{\nu}(x, f), x \in K$ as $\nu \rightarrow+\infty$ (see (3.1)). 
The series $\Phi_{i}(x, f), i=1,2$ have already been estimated in the proof of theorem 1.1, and the estimates (3.3) and (3.4), respectively, are true for them. It remains to estimate the series $\Phi_{3}(x, f)$ and $\Phi_{4}(x, f)$ under the conditions of theorem 1.2. Let $\alpha \in(1, \infty)$. Let us estimate the series $\Phi_{3}(x, f)$. By virtue of (3.8) and (3.9), to estimate $\Phi_{3}(x, f)$ it suffices to estimate the series

$$
\sum_{|\nu-| \lambda_{n}|| \geq 1,\left|\lambda_{n}\right| \geq 1}\left|\left(f, u_{n}\right)\right||\nu-| \lambda_{n}||^{-1 / \alpha^{\prime}}, \frac{1}{\alpha}+\frac{1}{\alpha^{\prime}}=1 .
$$

Let us show that the series $(3.13)$ is of order $O\left(\nu^{-\frac{1}{\alpha^{\prime}}}\right)$. Due to Corollary 2.1 (see $(2.10)$ ), for the series (3.13) we have

$$
\begin{aligned}
& \sum_{|\nu-| \lambda_{n}|| \geq 1,\left|\lambda_{n}\right| \geq 1}\left|\left(f, u_{n}\right)\right||\nu-| \lambda_{n}||^{-1 / \alpha^{\prime}} \leq \\
& \leq \sum_{|\nu-| \lambda_{n}|| \geq 1,\left|\lambda_{n}\right| \geq 1}\left|\left(B^{*} f^{\prime}, u_{n}\right)\right|\left|\lambda_{n}\right|^{-1}|\nu-| \lambda_{n}||^{-1 / \alpha^{\prime}}+ \\
& +\sum_{|\nu-| \lambda_{n}|| \geq 1,\left|\lambda_{n}\right| \geq 1}\left|\left(P f, u_{n}\right)\right|\left|\lambda_{n}\right|{ }^{-1}|\nu-| \lambda_{n}||^{-1 / \alpha^{\prime}} \leq \\
& \leq \sum_{\substack{1 \leq|\nu-| \lambda_{n} \| \leq \nu / 2 \\
\left|\lambda_{n}\right| \geq 1}}\left|\left(B^{*} f^{\prime}, u_{n}\right)\right|\left|\lambda_{n}\right|^{-1}|\nu-| \lambda_{n}||^{-1 / \alpha^{\prime}}+ \\
& +\left.\sum_{1 \leq\left|\lambda_{n}\right| \leq \nu / 2}\left|\left(B^{*} f^{\prime}, u_{n}\right)\right|\left|\lambda_{n}\right|^{-1}|\nu-| \lambda_{n}\right|^{-1 / \alpha^{\prime}}+ \\
& +\sum_{\left|\lambda_{n}\right| \geq 3 \nu / 2}\left|\left(B^{*} f^{\prime}, u_{n}\right)\right|\left|\lambda_{n}\right|^{-1}|\nu-| \lambda_{n}||^{-1 / \alpha^{\prime}}+ \\
& +\left.\sum_{\substack{1 \leq|\nu-| \lambda_{n} \| \leq \nu / 2 \\
\left|\lambda_{n}\right| \geq 1}}\left|\left(P f, u_{n}\right)\right|\left|\lambda_{n}\right|^{-1}|\nu-| \lambda_{n}\right|^{-1 / \alpha^{\prime}}+ \\
& +\sum_{1 \leq\left|\lambda_{n}\right| \leq \nu / 2}\left|\left(P f, u_{n}\right)\right|\left|\lambda_{n}\right|^{-1}|\nu-| \lambda_{n}||^{-1 / \alpha^{\prime}} \\
& +\left.\sum_{\left|\lambda_{n}\right| \geq 3 \nu / 2}\left|\left(P f, u_{n}\right)\right|\left|\lambda_{n}\right|^{-1}|\nu-| \lambda_{n}\right|^{-1 / \alpha^{\prime}} .
\end{aligned}
$$

As $f^{\prime}(x) \in L_{p}^{2}(G), p>1$, we have $B^{*} f^{\prime} \in L_{p}^{2}(G) \subset L_{\beta}^{2}(G)$, where $\beta=$ $\min \{p, 2\}$. Therefore, we can apply the Riesz inequality to the function $B^{*} f^{\prime}$ (see Lemma 2.5). Thus, for $\beta^{\prime}=\beta /(\beta-1)$ we have

$$
\left(\sum_{n=1}^{\infty}\left|\left(B^{*} f^{\prime}, u_{n}\right)\right|^{\beta^{\prime}}\right)^{1 / \beta^{\prime}} \leq M\left\|B^{*} f^{\prime}\right\|_{\beta, 2} .
$$

Applying Hölder's inequality and (3.15) to the first three sums on the righthand side of the inequality (3.14), we obtain

$$
\sum_{\substack{1 \leq|\nu-| \lambda_{n} \| \leq \nu / 2 \\\left|\lambda_{n}\right| \geq 1}}\left|\left(B^{*} f^{\prime}, u_{n}\right)\right|\left|\lambda_{n}\right|^{-1}|\nu-| \lambda_{n}||^{-1 / \alpha^{\prime}} \leq
$$




$$
\begin{aligned}
& \leq\left(\sum_{\substack{1 \leq|\nu-| \lambda_{n} \| \leq \nu / 2 \\
\left|\lambda_{n}\right| \geq 1}}\left|\left(B^{*} f^{\prime}, u_{n}\right)\right|^{\beta^{\prime}}\right)^{1 / \beta^{\prime}}\left(\sum_{\substack{1 \leq|\nu-| \lambda_{n} \| \leq \nu / 2 \\
\left|\lambda_{n}\right| \geq 1}}\left|\lambda_{n}\right|^{-\beta}|\nu-| \lambda_{n}||^{-\beta / \alpha^{\prime}}\right)^{1 / \beta} \leq \\
& \leq 2 M\left\|B^{*} f^{\prime}\right\|_{\beta, 2}\left(\sum_{1 \leq|\nu-| \lambda_{n}|| \leq \nu / 2}|\nu-| \lambda_{n}||^{-\beta / \alpha^{\prime}}\right)^{1 / \beta} \nu^{-1}= \\
& =O\left(\nu^{-1}\right)\left(\sum_{k=1}^{[\nu / 2]} k^{-\beta / \alpha^{\prime}}\left(\sum_{k \leq|\nu-| \lambda_{n}|| \leq k+1} 1\right)\right)^{1 / \beta}=O\left(\nu^{-1}\right)\left(\sum_{k=1}^{[\nu / 2]} k^{-\beta / \alpha^{\prime}}\right)^{1 / \beta}= \\
& =O\left(\nu^{-1}\right)\left\{\begin{array}{c}
\ln ^{1 / \beta} \nu \text { for } \beta(1-1 / \alpha)=1 \\
\nu^{1 / \beta-1 / \alpha^{\prime}} \text { for } \beta(1-1 / \alpha)<1 \\
1 \quad \text { for } \beta(1-1 / \alpha)>1
\end{array}\right. \\
& \sum_{1 \leq\left|\lambda_{n}\right| \leq \nu / 2}\left|\left(B^{*} f^{\prime}, u_{n}\right)\right|\left|\lambda_{n}\right|^{-1}|\nu-| \lambda_{n}\left\|^{-1 / \alpha^{\prime}} \leq M\right\| B^{*} f^{\prime} \|_{\beta, 2} \times \\
& \times\left(\left.\sum_{1 \leq\left|\lambda_{n}\right| \leq \nu / 2}\left|\lambda_{n}\right|^{-\beta}|\nu-| \lambda_{n}\right|^{-\beta / \alpha^{\prime}}\right)^{1 / \beta} \leq M\left\|B^{*} f^{\prime}\right\|_{\beta, 2} \nu^{-1 / \alpha^{\prime}} \times \\
& \times\left(\sum_{1 \leq\left|\lambda_{n}\right| \leq \nu / 2}\left|\lambda_{n}\right|^{-\beta}\right)^{1 / \beta}=O\left(\nu^{-1 / \alpha^{\prime}}\right) \\
& \left.\sum_{\left|\lambda_{n}\right| \geq 3 \nu / 2}\left|\left(B^{*} f^{\prime}, u_{n}\right)\right|\left|\lambda_{n}\right|^{-1}|\nu-| \lambda_{n}\right|^{-1 / \alpha^{\prime}} \leq M\left\|B^{*} f^{\prime}\right\|_{\beta, 2} \times \\
& \times\left(\left.\sum_{\left|\lambda_{n}\right| \geq 3 \nu / 2}\left|\lambda_{n}\right|^{-\beta}|\nu-| \lambda_{n}\right|^{-\beta / \alpha^{\prime}}\right)^{1 / \beta}=O\left(\nu^{-\beta\left(1+1 / \alpha^{\prime}\right)+1}\right)^{1 / \beta}= \\
& =O\left(\nu^{-1-1 / \alpha^{\prime}+1 / \beta}\right)=O\left(\nu^{-1 / \alpha^{\prime}}\right) .
\end{aligned}
$$

Consequently, the sum of the first three series on the right-hand side of (3.14) is of order $O\left(\nu^{-1 / \alpha^{\prime}}\right)$.

It can be similarly proved that the sum of the last three series in (3.14) is also of order $O\left(\nu^{-1 / \alpha^{\prime}}\right)$. In this case, as $\beta$ we need to take the number $\min \{2, \alpha\}$ and take into account that the vector function $P f$ belongs to $L_{\alpha}^{2}(G)$.

Thus, $\left\|\Phi_{3}(\cdot, f)\right\|_{C(K)}=O\left(\nu^{-1 / \alpha^{\prime}}\right)$ as $\nu \rightarrow+\infty$. This estimate is also true for $\Phi_{4}(x, f)$.

Consequently, for $\alpha \in(1, \infty)$ we have the estimate

$$
\left\|\sigma_{\nu}(\cdot, f)-\tilde{S}_{\nu}(\cdot, f)\right\|_{C(K)}=O\left(\nu^{-1 / \alpha^{\prime}}\right), \nu \rightarrow+\infty
$$

Now we consider the case $\alpha=\infty$ and estimate the series $\Phi_{3}(x, f)$ in the metrics $C(K)$ (the series $\Phi_{4}(x, f)$ is estimated in a similar way). For $\Phi_{3}(x, f)$, 
when $\alpha=\infty$, we already have the inequality (3.11). Therefore, it suffices to estimate the series

$$
\sum_{|\nu-| \lambda_{n}|| \geq 1}\left|\left(f, u_{n}\right)\right||\nu-| \lambda_{n}||^{-1} \ln \left(1+|\nu-| \lambda_{n}||\right) .
$$

Let us prove that this series is of order $O\left(\nu^{-1} \ln \nu\right)$. The sum of terms corresponding to the eigenvalues $\lambda_{n}$ with $\left|\lambda_{n}\right|<1$ is of order $O\left(\nu^{-1} \ln \nu\right)$ (see the proof of theorem 1.1 for $\alpha=\infty$ ). For $\left|\lambda_{n}\right| \geq 1$ we apply Corollary 2.1 (see (2.10)). Then we have

$$
\begin{aligned}
& \sum_{\substack{|\nu-| \lambda_{n} \| \geq 1 \\
\left|\lambda_{n}\right| \geq 1}}\left|\left(f, u_{n}\right)\right||\nu-| \lambda_{n}||^{-1} \ln \left(1+|\nu-| \lambda_{n}||\right) \leq \\
& \leq \sum_{\substack{|\nu-| \lambda_{n}|| \geq 1 \\
\left|\lambda_{n}\right| \geq 1}}\left|\left(B^{*} f^{\prime}, u_{n}\right)\right| \frac{\ln \left(1+|\nu-| \lambda_{n}||\right)}{\left|\lambda_{n}\right||\nu-| \lambda_{n}||}+ \\
& +\sum_{\substack{|\nu-| \lambda_{n}|| \geq 1 \\
\left|\lambda_{n}\right| \geq 1}}\left|\left(P f, u_{n}\right)\right| \frac{\ln \left(1+|\nu-| \lambda_{n}||\right)}{\left|\lambda_{n}\right||\nu-| \lambda_{n}||} \leq \\
& \leq \sum_{1 \leq|\nu-| \lambda_{n}|| \leq \nu / 2,\left|\lambda_{n}\right| \geq 1}\left|\left(B^{*} f^{\prime}, u_{n}\right)\right| \frac{\ln \left(1+|\nu-| \lambda_{n}||\right)}{\left|\lambda_{n}\right||\nu-| \lambda_{n}||}+ \\
& +\sum_{1 \leq\left|\lambda_{n}\right| \leq \nu / 2}\left|\left(B^{*} f^{\prime}, u_{n}\right)\right| \frac{\ln \left(1+|\nu-| \lambda_{n}||\right)}{\left|\lambda_{n}\right||\nu-| \lambda_{n}||}+ \\
& +\sum_{\left|\lambda_{n}\right| \geq 3 \nu / 2}\left|\left(B^{*} f^{\prime}, u_{n}\right)\right| \frac{\ln \left(1+|\nu-| \lambda_{n}||\right)}{\left|\lambda_{n}\right||\nu-| \lambda_{n}||}+ \\
& +\sum_{1 \leq|\nu-| \lambda_{n}|| \leq \nu / 2,\left|\lambda_{n}\right| \geq 1}\left|\left(P f, u_{n}\right)\right| \frac{\ln \left(1+|\nu-| \lambda_{n}||\right)}{\left|\lambda_{n}\right||\nu-| \lambda_{n}||}+ \\
& +\sum_{1 \leq\left|\lambda_{n}\right| \leq \nu / 2}\left|\left(P f, u_{n}\right)\right| \frac{\ln \left(1+|\nu-| \lambda_{n}||\right)}{\left|\lambda_{n}\right||\nu-| \lambda_{n}||}+ \\
& +\sum_{\left|\lambda_{n}\right| \geq 3 \nu / 2}\left|\left(P f, u_{n}\right)\right| \frac{\ln \left(1+|\nu-| \lambda_{n}||\right)}{\left|\lambda_{n}\right||\nu-| \lambda_{n}||} .
\end{aligned}
$$

The vector functions $B^{*} f^{\prime}$ and $P f$ belong to the spaces $L_{p}^{2}(G)$ and $L_{2}^{2}(G)$, respectively, because $f(x) \in W_{p, 2}^{1}(G), p>1$, and $p(x), q(x) \in L_{\infty}(G)$. Therefore, for the vector function $B^{*} f^{\prime}$ we can apply the Riesz inequality with $\gamma=$ $\max \{2, q\}, q=\frac{p}{(p-1)}$, and for the vector function $P f$ we can apply Bessel's inequality, i.e. the Riesz inequality with $\gamma=2$ (see lemma 2.5). Then, by virtue of the Hölder's, Riesz inequalities and (2.4), from (3.16) we obtain $\left(\gamma^{-1}+\left(\gamma^{\prime}\right)^{-1}=1\right)$ :

$$
\sum_{\substack{|\nu-| \lambda_{n}|| \geq 1 \\\left|\lambda_{n}\right| \geq 1}}\left|\left(f, u_{n}\right)\right| \frac{\ln \left(1+|\nu-| \lambda_{n}||\right)}{|\nu-| \lambda_{n}||} \leq
$$




$$
\begin{aligned}
& \leq M_{\gamma^{\prime}}\left\|B^{*} f^{\prime}\right\|_{\gamma^{\prime}, 2}\left\{\left(\sum_{\substack{1 \leq|\nu-| \lambda_{n}|| \leq \nu / 2 \\
\left|\lambda_{n}\right| \geq 1}} \frac{\ln ^{\gamma^{\prime}}\left(1+|\nu-| \lambda_{n}||\right)}{\left|\lambda_{n}\right|^{\gamma^{\prime}}|\nu-| \lambda_{n}||^{\gamma^{\prime}}}\right)^{1 / \gamma^{\prime}}+\right. \\
& \left.+\left(\sum_{1 \leq\left|\lambda_{n}\right| \leq \nu / 2} \frac{\ln ^{\gamma^{\prime}}\left(1+|\nu-| \lambda_{n}||\right)}{\left|\lambda_{n}\right|^{\gamma^{\prime}}|\nu-| \lambda_{n}||^{\gamma^{\prime}}}\right)^{1 / \gamma^{\prime}}+\left(\sum_{\left|\lambda_{n}\right| \geq 3 \nu / 2} \frac{\ln ^{\gamma^{\prime}}\left(1+|\nu-| \lambda_{n}||\right)}{\left|\lambda_{n}\right|^{\gamma^{\prime}}|\nu-| \lambda_{n}||^{\gamma^{\prime}}}\right)^{1 / \gamma^{\prime}}\right\}+ \\
& +M_{2}\|P f\|_{2,2}\left\{\left(\sum_{\substack{1 \leq|\nu-| \lambda_{n}|| \leq \nu / 2 \\
\left|\lambda_{n}\right| \geq 1}} \frac{\ln ^{2}\left(1+|\nu-| \lambda_{n}||\right)}{\left.\left|\lambda_{n}\right|^{2}|\nu-| \lambda_{n}\right|^{2}}\right)^{1 / 2}+\right. \\
& \left.+\left(\sum_{1 \leq\left|\lambda_{n}\right| \leq \nu / 2} \frac{\ln ^{2}\left(1+|\nu-| \lambda_{n}||\right)}{\left.\left|\lambda_{n}\right|^{2}|\nu-| \lambda_{n}\right|^{2}}\right)^{1 / 2}+\left(\sum_{\left|\lambda_{n}\right| \geq 3 \nu / 2} \frac{\ln ^{2}\left(1+|\nu-| \lambda_{n}||\right)}{\left.\left|\lambda_{n}\right|^{2}|\nu-| \lambda_{n}\right|^{1 / 2}}\right)^{1 / 2}\right\} \leq \\
& \leq M_{\gamma^{\prime}}\left\|B^{*} f^{\prime}\right\|_{\gamma^{\prime}, 2}\left\{\frac{2 \ln (1+\nu)}{\nu}\left(\sum_{k=1}^{[\nu / 2]} \frac{1}{k^{\prime}}\left(\sum_{k \leq|\nu-| \lambda_{n}|| \leq k+1} 1\right)\right)^{1 / \gamma^{\prime}}+\right. \\
& +\frac{2 \ln \nu}{\nu}\left(\sum_{k=1}^{[\nu / 2]} \frac{1}{k^{\gamma^{\prime}}}\left(\sum_{k \leq\left|\lambda_{n}\right| \leq k+1} 1\right)\right)^{1 / \gamma^{\prime}}+ \\
& \left.+\left(\sum_{k=[\nu / 2]}^{\infty} \frac{\ln ^{\gamma^{\prime}}(2+k)}{k^{2 \gamma^{\prime}}}\left(\sum_{k \leq|\nu-| \lambda_{n}|| \leq k+1} 1\right)\right)^{1 / \gamma^{\prime}}\right\}+ \\
& +M_{2}\|P f\|_{2,2}\left\{\frac{2 \ln (1+\nu)}{\nu}\left(\sum_{k=1}^{[\nu / 2]} \frac{1}{k^{2}}\left(\sum_{k \leq|\nu-| \lambda_{n}|| \leq k+1} 1\right)\right)^{1 / 2}+\right. \\
& +\frac{2 \ln \nu}{\nu}\left(\sum_{k=1}^{[\nu / 2]} \frac{1}{k^{2}}\left(\sum_{k \leq\left|\lambda_{n}\right| \leq k+1} 1\right)\right)^{1 / 2}+ \\
& \left.+\left(\sum_{k=[\nu / 2]}^{\infty} \frac{\ln ^{2}(2+k)}{k^{4}}\left(\sum_{k \leq|\nu-| \lambda_{n}|| \leq k+1} 1\right)\right)^{1 / 2}\right\}=O\left(\frac{\ln \nu}{\nu}\right) .
\end{aligned}
$$

Consequently, the estimate $\left\|\Phi_{i}(\cdot, f)\right\|_{C(K)}=O\left(\nu^{-1} \ln \nu\right), i=3,4, \nu \rightarrow+\infty$ holds for $\alpha=\infty$. Combining this with the estimates (3.3) and (3.4), from (3.1) we get

$$
\left\|\tilde{S}_{\nu}(\cdot, f)-\sigma_{\nu}(\cdot, f)\right\|_{C(K)}=O\left(\nu^{-1} \ln \nu\right), \nu \rightarrow+\infty .
$$

Theorem 1.2 is completely proved. 


\section{References}

[1] A.M. Abdullayeva, On local componentwise equiconvergence for one-dimensional Dirac operator. Trans. Natl. Acad. Sci. Azerb. Ser. Phys.-Tech. Math. Sci., 39 (2019), no.1, Mathematics, 3-14.

[2] V.A. Il'in, Necessary and sufficient conditions for basicity and equiconvergence of spectral expansions with trigonometric series I. Diff. Uravn., 16 (1980), no.5, 771794.

[3] V.A. Il'in, Necessary and sufficient conditions for basicity and equiconvergence of spectral expansions with trigonometric series II. Diff. Uravn., 16 (1980), no.6, 9801009.

[4] V.A. Il'in, Componentwise equiconvergence with trigonometric series of expansions into root vector functions of Schrödinger operator with non-Hermitian matrix potential, whose elements are only summable. Diff. Uravn., 27 (1991), no.11, 1862-1878.

[5] V.M. Kurbanov, Equiconvergence theorem for differential operators with locally summable coefficients. Trudy IMM AN Azerb., 6 (1996), 168-174.

[6] V.M. Kurbanov, On equiconvergence rate of spectral expansions. Dokl. RAN, $\mathbf{3 6 5}$ (1999), no.4, 444-449.

[7] V.M. Kurbanov, A.I. Ismailova, Properties of root vector functions of onedimensional Dirac operator. Dokl. RAN, 433 (2010), no.6, 736-740.

[8] V.M. Kurbanov, A.I. Ismailova, Componentwise uniform equiconvergence of expansions into root vector functions of Dirac operator with trigonometric expansion. Diff. Uravn., 48 (2012), no.5, 648-662.

[9] V.M. Kurbanov, On Bessel property and unconditional basicity of the system of root vector-functions of the Dirac operator. Diff. Uravn., 32 (1996), no.12, 1608-1617.

[10] V.M. Kurbanov, A.I. Ismailova, Two-sided estimates for root vector functions of Dirac operator. Diff. Uravn., 48 (2012), no.4, 487-497.

[11] V.M. Kurbanov, A.M. Abdullayeva, Bessel property and basicity of the system of root vector functions of Dirac operator with summable coefficient. Operators and Matrics, 2018, 12 (2018), no.4, 443-454.

[12] V.M. Kurbanov, Equiconvergence of biorthogonal expansions in root functions of differential operators II. Differ. Equations, 36 (2000), no.3, 319-335.

[13] A. Zygmund, Trigonometric series vol. 2, Camrige; Cambrige Univ. Press, 1960, Translated under the title Trigonometricheskie ryady, 2 (1965), Moscow: Mir.

Vali M. Kurbanov

Institute of Mathematics and Mechanics of NAS of Azerbaijan, Azerbaijan State Pedagogical University

E-mail address: q.vali@yahoo.com

Afsana M. Abdullayeva

Azerbaijan State Pedagogical University

E-mail address: ef .abdullayeva@inbox.ru

Received: July 15, 2019; Accepted: October 22, 2019 